\title{
Neonatal outcome following buprenorphine maintenance for opiate dependency
}

\author{
John Fagan, Eamon Keenan
}

Ir J Psych Med 2008; 25(4): 141-144

\begin{abstract}
Methadone substitution improves maternal and neonatal outcomes. However methadone induced neonatal abstinence syndrome (NAS) is common. Buprenorphineexposed neonates may be at a lower risk of NAS. Currently in the Republic of Ireland, buprenorphine does not have a special licence for use in pregnancy. We describe here the history and neonatal outcomes of the first Irish woman maintained on buprenorphine during two pregnancies.

Supervised urinanalysis on this mother between and throughout both pregnancies did not reveal any illicit drug use. She delivered two post-term babies of normal birth weight and length. The second infant required treatment for NAS for 21 days with morphine sulphate. Although the use of buprenorphine in pregnancy does not remove the possibility of NAS, neonatal outcomes of buprenorphinemaintained women compares favourably to methadone. As the use of buprenorphine becomes more established in Ireland, the management of buprenorphine-exposed neonates will become more common.
\end{abstract}

Key words: Neonatal abstinence syndrome; Opiate dependency; Buprenorphine.

\section{Introduction}

The incidence of heroin use in Ireland escalated throughout the 1990s before tailing off at the end of the decade, ${ }^{1}$ only to increase again in recent years. ${ }^{2}$ However, reflecting the chronic nature of opiate dependency, prevalence rates have steadily increased. Recent estimates of opiate prevalence in Ireland are based on a three-source capturerecapture study of opiate use in 2000-2001. ${ }^{3}$

This research showed that in the 15-64 year age group, $5.6 / 1000$ of the population used opiates; local prevalence in the Dublin area was amongst the highest in Europe at 15.9/1000. In the 15-24 year age group, prevalence estimates were even higher at $32 / 1000$ males and $17 / 1000$ females.

Increasingly more women are entering methadone substitution treatment, the majority of childbearing age. Internationally and in Ireland, methadone, a pure opiate

*John Fagan, Psychiatric Registrar, YoDA Addiction service, Glen Abbey Building, Belgard Road, Dublin 24, Ireland. Email: johnc.fagan@hse.ie

Eamon Keenan, Consultant Psychiatrist, Addiction Services, Bridge House, Cherry Orchard Hospital, Ballyfermot, Dublin 10, Ireland. Health Service Executive - Dublin Mid Leinster, Ireland. *Correspondence

SUBMITTED: FEBRUARY 18, 2008. ACCEPTED: MAY 19, 2008. agonist, is the main substitution medication used in the treatment of opiate dependency due to its proven ability to stabilise the opiate-dependent person's drug use and lifestyle. ${ }^{4}$ Methadone facilitates engaging and retaining an opiate-dependent individual in treatment so that at the very least, they can be offered medical, psychological and social support ('harm reduction'). For methadone-maintained mothers, treatment facilitates access to antenatal and obstetric care, which can be co-ordinated by a drug liaison midwife.

In 1999, a drug liaison midwife service was set up with one midwife attached to each of the three Dublin Maternity Hospitals. In the Republic of Ireland during 2005, there were 8,962 people prescribed methadone, ${ }^{5}$ of whom 2,746 were female $(30.6 \%)$, with a mean age of 29 years (SD +/- $6.4 \mathrm{yrs})$. And during that year, there were 193 infants born in the three Dublin maternity hospitals to women maintained on methadone. ${ }^{6}$

Research has consistently demonstrated that maternal and neonatal outcomes are better in methadone-maintained mothers than for those mothers using opiates and not in treatment. $^{7-10}$ However, a neonatal abstinence syndrome (NAS) occurs in 48-94\% of infants exposed to methadone in-utero. ${ }^{11}$

The signs of NAS include: high-pitched cry, rapid breathing, hungry but ineffective sucking, excessive wakefulness, tremor, vomiting and diarrhoea, hypertonicity, sweating, and rarely, seizures. In the interest of objectivity, Loretta Finnegan and colleagues developed the 'Finnegan chart' which allows the assessment and monitoring of NAS. ${ }^{12}$ Numerical values are allocated on the basis of the presence and severity of 20 signs commonly found in neonatal withdrawal (CNS, metabolic, vasomotor, respiratory, and gastro intestinal disturbances). The numbers are added together to give a total score (maximum 46).

The aims of managing an infant who is at risk of NAS are to maintain a normal temperature, to ensure adequate sleep between feeds, to reduce hyperactivity and excessive crying, to reduce motor instability, and ensure adequate weight gain. The hospital where these infants were delivered uses the 'Liverpool approach'17 (an obstetric liaison service comprising of a multidisciplinary team from the addiction and midwifery services), and monitors for NAS with a modified version of the Finnegan Scale.

The decision to initiate treatment of opiate withdrawal with morphine sulphate is made if any one score is greater than 12 , or the infant scores eight or more on three successive occasions.

The dose and schedule of morphine sulphate substitution is adjusted so as to maintain the daily Finnegan scores under eight, and then gradually reduced as the infant shows evidence of prospering. 
While research from our own service indicated that higher maternal methadone doses are associated with increased likelihood of NAS, ${ }^{13}$ debate on this issue exists in the literature..$^{14,15}$ When NAS occurs, how long it lasts and how severe it becomes depends on many factors including other drugs used, the amounts used, when these were taken in relation to delivery, and the condition of the infant after birth. ${ }^{16}$ As a result of NAS, infants of methadone-maintained mothers may require prolonged hospitalisation, ${ }^{17,18}$ which in addition to disrupting family bonding, increases the costs to service providers.

Since the mid 1990s, buprenorphine (Subutex), a mixed agonist-antagonist has become increasingly available in Europe as an alternative to methadone. In 2002, legislative changes in Ireland allowed buprenorphine to be prescribed to non-pregnant people requiring substitution treatment. Whereas methadone is a full agonist at the mu-opiate receptor, and as formulated for use in Ireland, cannot be injected, buprenorphine is a partial agonist at the mu-receptor, an antagonist at the K-opiate receptor, and can be injected.

Research on adults suggests that buprenorphine may be easier to withdraw from than methadone. ${ }^{19}$ In addition buprenorphine may have advantages over methadone in pregnancy, as the incidence and severity of NAS appears to be less ${ }^{20-24}$ resulting in less distress to the infant and mother, and shorter hospital admissions. This may reflect the different receptor profile of buprenorphine. ${ }^{25}$

More definitive evidence may come from the 'Mother Opioid Treatment: Human Experimental Research' project (MOTHER), the first large, multicentre, randomised, doubleblind study to compare methadone and buprenorphine for the treatment of pregnant opiate-dependent pregnant women and the effect on the NAS. ${ }^{26}$ Preliminary data suggests that buprenorphine results in improved birth outcomes and less neonatal abstinence syndrome relative to methadone. ${ }^{27,28}$

In addition, whereas breast-fed infants of mothers who continue on methadone have been found to have less severe NAS and require as a result to be weaned off breast-feeds, ${ }^{29}$ buprenorphine levels in breast milk have little or no effect on the NAS. ${ }^{30}$ However as buprenorphine can be injected some argue that this undermines one of the main benefits of methadone, which is reducing injecting behaviour and the spread of blood-borne diseases.

To counter this abuse potential, buprenorphine has been combined with naloxone (Suboxone) in a 4:1 ratio. Naloxone, a pure opiate competitive antagonist with good parenteral but poor oral/sublingual bioavailability, will precipitate opiate withdrawal in opiate-dependent individuals when injected - this aversive effect lessens the risk of diversion and intravenous misuse. As a result, this combined buprenorphine preparation is likely to be the main alternative to methadone in Ireland.

Whilst teratology studies on animals reveal no embryogenic/teratotogenic effects associated with methadone or buprenorphine, there are concerns of reproductive toxicity related to naloxone. ${ }^{31}$ Therefore women maintained on buprenorphine/naloxone who become pregnant should be switched over to buprenorphine when pregnancy is confirmed. . $^{31,32}$

As the use of buprenorphine preparations to treat opiatedependence becomes more established in Ireland, conception and pregnancy while on buprenorphine treatment and the subsequent management of such pregnancies will also become more frequent.

\section{Case report}

Ms A grew up in a family with no known history of alcohol/substance misuse or psychiatric illness. She left school when she was 16 years old after passing the Junior Certificate and began working. Through associating with an older age group, she was introduced to illegal drugs. She first used heroin when she was 16 years old ('skin-popping') and quickly progressed to daily intravenous use of heroin (and cocaine). Soon she had moved out of the family home to become homeless. With the future father of her children, she spent three periods living homeless in a city in England.

During the first visit in 2000 , she developed a femoral vein thrombosis, and commenced methadone maintenance treatment for the first time. On returning to Ireland, she completed a three-month outpatient detoxification, but relapsed and went back to England with her boyfriend. After several months of chaotic drug use, she self-detoxed and returned to Ireland, where she remained abstinent for about 18 months.

In 2004, she and her boyfriend returned to England for the last time. Again they lived a homeless drug-dependent lifestyle until Ms A was identified and engaged into treatment. During the assessment process, she discovered she was pregnant (and positive for hepatitis $\mathrm{C}$ ).

Instead of methadone, she chose buprenorphine substitution treatment, and stabilised on $12 \mathrm{mg}$. Emergency accommodation was arranged for her but not her partner, as he did not enter treatment, and subsequently they lost contact. Late in her pregnancy, Ms A decided to return to live with her parents in Dublin. In January 2005 as arranged, she presented for treatment to the Drug Treatment Centre Board. She was 21 years old and 36 weeks pregnant.

A decision was made to continue buprenorphine rather than switch to methadone due to her imminent delivery. Ms A was assigned a drug liaison midwife and linked in with the obstetric services.

Her urines were closely monitored and remained negative for all drugs of abuse. One month later she gave birth to a healthy baby. A few weeks later, on release from prison, her partner returned to live with her in Ireland.

Ms A continued buprenorphine maintenance treatment following the birth of her baby and became pregnant again after a few months. Early in her second pregnancy, Ms A complained of symptoms that she associated with opiate withdrawal. These settled on increasing the dose of buprenorphine incrementally to $16 \mathrm{mg}$.

Late in this second pregnancy, her partner returned to England and unfortunately died from a brain haemorrhage. Ms A moved back to live with her parents, did not relapse and continued to give clean urines. She gave birth to her second child in early 2006. During both pregnancies, she abstained from alcohol, and tried to smoke less than 10 cigarettes per day.

\section{Neonatal outcomes}

Table 1 compares the birth parameters of these two infants. Both of this woman's pregnancies were without complication. Both infants were delivered post-term, and 


\section{Table 1: Birth parameter comparison for baby one and baby two}

Characteristic
Mother's weight at booking
Exposed to buprenorphine
Buprenorphine dose at delivery
Antenatal ultrasound
Gestational age
Delivered by
Sex of infant
Weight of infant
Lowest weight
Length of infant (crown-heel)
Head circumference (32-37 cm)
Apgar score 1, 5 minutes
Maximum NAS score
Length of stay in Neonatal ICU
Length of substitution treatment
Total length of hospital stay
Six week check-up

\section{Baby one}

Primagravida

Not known

After conception

$12 \mathrm{mg}$ once daily

Normal

$41+1$

Spontaneous vaginal delivery

Female

$3.24 \mathrm{~kg}$

$2.89 \mathrm{~kg}$

$49 \mathrm{~cm}$

$35 \mathrm{~cm}$

9,10

$9(\mathrm{x} 2)$

Not admitted

Not initiated

5 days

Normal - weight $4.57 \mathrm{~kg}$

Head circum $37 \mathrm{~cm}$
Baby two

Para $1+0$

$52 \mathrm{~kg}$

At conception (12mg)

$16 \mathrm{mg}$ once daily

Normal

42

Induced vaginal delivery

Male

$3.6 \mathrm{~kg}$

$3.24 \mathrm{~kg}$

$51 \mathrm{~cm}$

$35 \mathrm{~cm}$

9,10

16

29 days

21 days - day 2 to day 23

30 days - ready for d/c D26

Normal - weight $4.71 \mathrm{~kg}$

Head circum $38 \mathrm{~cm}$ were healthy at delivery, with normal birth weights for gestational age relative to the mother's height $(157 \mathrm{~cm})$ and weight. Neurodevelopmental examinations at six months were also normal. Neither infant was breastfed.

\section{Baby one}

This infant girl was a spontaneous non-instrumental vertexvaginal delivery, during which the mother did not require analgesia. She developed mild symptoms of neonatal abstinence in the first day, with a maximum Finnegan score of nine on two occasions. These scores reflected mild irritability overnight due to mild tremors, sneezing, loose stools and secondary excoriation of the buttock area. Treatment for NAS was not required. By day five she was gaining weight, and was ready for discharge with her mother. The baby's weight on discharge was $2.84 \mathrm{~kg}$, and two weeks later was $3.25 \mathrm{~kg}$. At this check-up, the mother reported very occasional sweating episodes but no jitteriness.

\section{Baby two}

In contrast, baby two was induced at two weeks post-term. This delivery was more distressing for the mother, as on this occasion she required pethidine analgesia. The third stage of delivery took 35 minutes. The placental cord was around this infant's neck, but the baby was not distressed, cried at delivery, and Apgar scores were normal. Mild but significant symptoms of NAS developed within the first 24 hours (Finnegan Scores 6, 5).

On day two, this baby boy was admitted to the neonatal intensive care unit in order to facilitate the mother attending her partner's cremation. The mother was subsequently discharged on day five.

Symptoms of NAS continued to increase during the second day $(8,4,11)$ peaking at 15 by 42 hours, at which time substitution treatment with morphine sulphate was initiated $(0.2 \mathrm{mg}$ every three hours). The dose was gradually reduced, and the interval between doses slowly extended, so that by day nine the infant was stable on $0.1 \mathrm{mg}$ every six hours and had reached a lowest weight of $3.24 \mathrm{~kg}$. With a gain of $10 \mathrm{~g}$ by day 10 , the interval was extended to eighthourly, however the infant became distressed, but settled again on six-hourly doses.

During the next few days this regime was continued whilst the baby continued to put on weight. Detoxification was recommenced at day 16 and successfully completed on day 23. The total duration of treatment with morphine sulphate was 21 days. This period of detoxification was also complicated by a pseudomonas eye infection treated with topical gentamycin from day six, and augmented with fucidic acid from day 25. The infant was ready for discharge on day 26 (weight $3.84 \mathrm{~kg}$ ), but this was deferred until day 30 for nonmedical reasons (weight $4.10 \mathrm{~kg}$ ).

\section{Discussion}

This unique case history describes the neonatal outcomes of two infants delivered to a mother who successfully stabilised on buprenorphine substitution therapy. Unlike baby one, baby two had a prolonged hospital stay as a result of significant NAS. The duration of treatment was similar to the mean treatment period shown in a previous Irish study by 
Coghlan and colleagues in the same maternity hospital. ${ }^{18}$ This study showed that the mean duration of treatment for an infant with NAS was 21.8 days (range 1-62 days); however, only six of the 43 infants reviewed were exposed to methadone alone, with a mean duration of treatment of 17 days.

The widely diverse degree of NAS between these two infants and the documented history of illicit drug abstinence by their mother, make it easier to appreciate that even in such ideal circumstances, it is not possible to predict the likelihood and degree of NAS a newborn might experience. Other factors must contribute to opiate related NAS, an area warranting further research.

Possible other factors contributing to the severity of NAS in baby two's case might include:

- Exposure to buprenorphine from conception

- As with methadone, the possibility of a dose effect. Early pregnancy symptoms can mimic withdrawal, ${ }^{17}$ and this may explain the mother's need for an increased dose in the first trimester

- Six days older at delivery - pre-term babies appear to be less effected by NAS, and reasons postulated include a shorter duration of exposure to drugs, and a less mature nervous system ${ }^{33}$

- Labour was induced

- Pethidine was administered which may have contributed to opiate withdrawal

- Psychosocial stress factors related to the sudden death of the mothers partner. Perhaps she smoked more as a result of this unexpected event. Research has shown that nicotine can contribute to NAS in methadone-exposed neonates ${ }^{34}$

- Did urine drug toxicology accurately reflect abstinence from illicit drugs? New clients to the addiction services initially provide twice weekly, and later weekly, random urine samples, which are tested for the presence of opiates, methadone, benzodiazepines, cocaine, amphetamine, cannabis and alcohol. Short acting non-benzodiazepine sedatives are not routinely screened for. However the duration of negative toxicology suggests that she was truly abstinent.

\section{Conclusion}

Buprenorphine treatment for pregnant opiate-dependent women compares favourably to methadone. As this case shows, widely diverse neonatal outcomes can still be expected with buprenorphine substitution in pregnancy even when only exposed to buprenorphine in-utero. As the use of buprenorphine preparations for substitution and maintenance becomes more established in Ireland, the need to manage buprenorphine-exposed neonates will become more common.

Declaration of Interest: None.
References

1. Smyth BP, O'Brien M. Children Attending Addiction Treatment Services in Dublin 1990-1999. Eur Addict Res 2004; 10: 68-74.

2. European Monitoring Centre for Drugs and Drug Addiction (EMCDDA). 2002 Annual report on the state of the drugs problem in the European Union and Norway. Luxembourg: Office for Official Publications of the European Communities, 2002.

3. Kelly A, Carvallo M, Teljeur C. Prevalence of opiate use in Ireland 2000-2001. A 3source Capture Recapture study, NACD 2003.

4. Farrell M, Ward J, Mattick R et al. Methadone maintenance treatment in opiate dependence: a review. BMJ 1994; 309: 997-1001.

5. Central Methadone Treatment List, Drug Treatment Central Board, 30-31 Pearse Street, Dublin 2.

6. Personal correspondence, Drug Liaison Midwives.

7. Finnegan LP. Treatment issues for opioid-dependant women during the perinatal period. J Psychoactive Drugs 1991; 23; 191-201.

8. Fischer G. Treatment of opioid dependence in pregnant women. Addiction 2000; 95 : 1141-44.

9. Ward J, Mattick RP, Hall W. Methadone maintenance during pregnancy. In: Ward J, Mattick RP, Hall W eds. Methadone Maintenance Treatment and Other Opioid Replacement Therapies. Amsterdam: Harwood Academic Publishers 1998.

10. Hulse G, Milne E, English DR, Holman CDJ. The relationship between maternal use of heroin and methadone and infant birth weight. Addiction 1997; 92: 1571-1580.

11. Osborn DA, Cole MJ, Jeffrey HE. Opiate treatment for opiate withdrawal in newborn infants. Cochrane Library 2004, issue 3. Oxford: Update Software.

12. Finnegan LP, Kron RE, Connaughton JF, Emich JP. Assessment and treatment of abstinence in the infant of the drug dependent mother. Int J Clin Pharmacol 1975; 12: 19-32.

13. Scully M, Geoghegan N, Corcoran P, Tiernan M, Keenan E. Specialized drug liaison midwife services for pregnant opioid dependent women in Dublin, Ireland. J Subst Abuse Treatm 2004; 26: 329-335.

14. Dashe JS, Sheffield JS, Olscher DA, Todd SJ, Jackson GL, Wendel GD. Relationship between maternal methadone dosage and neonatal withdrawal. Obstet Gynecol 2002; 100(6): 1244-1249.

15. Stimmel B, Goldberg J, Reisman A, Murphy RJ, Teets K. Fetal outcome in narcoticdependent women: the importance of the type of maternal narcotic used. Am J Drug Alcohol Abuse 1982-83; 9(4): 383-395.

16. Morrison CL, Siney C. A survey of the management of neonatal opiate withdrawal. Eur J Paediatrics 1996; 155: 323-326.

17. Johnson A, Greenough A, Gerada C. Maternal drug use and length of neonatal unit stay. Addiction 2002; 98:785-789.

18. Coghlan D, Milner M, Clarke T et al. Neonatal abstinence syndrome. Ir Med J 1999; 92(1): 232-233, 236

19. Seifert J, Metzner C, Paetzold W et al. Detoxification of opiate addicts with multiple drug use: a comparison of buprenorphine vs. methadone. Pharmacopsychiatry 2002; 35: $159-164$.

20. Jones $\mathrm{HE}$, Johnson RE, Jasinski DR et al. Buprenorphine versus methadone in the treatment of pregnant opioid-dependent patients: effects on the neonatal abstinence syndrome. Drug Alcohol Depend 2005; 79: 1-10.

21. Fischer G, Johnson RE, Eder H et al. Treatment of opioid-dependent pregnant women with buprenorphine. Addiction 2000; 95(2): 239-244.

22. Lacroix I, Berrebi A, Chaumerliac C, Lapeyre-Mestre M, Montastruc JL, DamaseMichel C. Buprenorphine in pregnant opioid-dependent women: first results of a prospective study. Addiction 2004; 99: 209-214.

23. Schindler SD, Eder H, Ortner R, Rohrmeister K, Langer M, Fischer G. Neonatal outcome following buprenorphine maintenance during conception and throughout pregnancy. Addiction 2003; 98: 103-110.

24. Johnson RE, Jones HE, Fischer G. Use of buprenorphine in pregnancy: patient management and effects on the neonate. Drug Alcohol Depend 2003; 70(2 Suppl): S87-S101.

25. Lewis J, Rance MJ, Sanger DJ. The Pharmacology and abuse potential of buprenorphine: a new antagonistic analgesic. Adv Subt Abuse 1983; 3: 103-154.

26. Jones HE, Martin PR, Heil SH et al. Treatment of opioid-dependent Pregnant Women: Clinical and Research Issues. J Subst Abuse Treat in Press (available on-line) 27. Fischer G, Ortner R, Rohrmeister K et al. Methadone versus buprenorphine in pregnant addicts: a double-blind, double-dummy comparison study. Addiction 2006; 101: 275-281

28. NIDA - Directors Report - February 2005. www.nida.nih.gov/DirReports/ DirRep205/DirectorsReport13.html

29. Abdel-Latif ME, Pinner J, Clews S, Cooke F, Lui K, Oei J. Effects of breast milk on the severity and outcome of neonatal abstinence syndrome among infants of drugdependent mothers. Pediatrics 2006: 117: 1163-69.

30. Auriacombe M, Loustauneau A. Medical treatment of the pregnant heroin addict Review of the literature. In: Pregnancy and drug misuse update 2000. Proceedings: Seminar organised by the Co-operation Group to Combat Drug Abuse and Illicit Trafficking in Drugs (Pompidou Group). Drugs and Addiction. Council of Europe (2000), 39-74.

31. Summary of Product Characteristics (SPC) from the IPHA Electronic Medicines Compendium. www.medicines.ie

32. Centre for Substance abuse treatment. Medication-assisted treatment for opioid addiction in opioid treatment programs. US Department of Health and Human Services, Rockvill, MD (2005).

33. American Academy of Pediatrics Committee on Drugs. Neonatal drug withdrawal. Pediatrics 1998; 101: 1079-1085.

34. Choo RE, Huestis MA, Schroeder JR, Shin AS, Jones HE. Neonatal abstinence syndrome in methadone-exposed infants is altered by level of prenatal tobacco exposure. Drug Alcohol Depend 2004; 75: 253-260. 